\title{
Fecal Streptococci Producing Glycosyltransferase
}

\author{
Akira Inoue, Kazunaga Yazawa and Kiyosi Kondo \\ Sagami Chemical Research Center, Sagamihara, Kanagawa 229
}

(Received for publication, March 9, 1990)

\begin{abstract}
Streptococcal strains producing glycosyltransferase (GT) were surveyed from 15 fecal samples collected from 13 humans. In total, 219 strains showing marked mucoidicity were detected on the sucrose-containing Brain Heart Infusion plate medium. Twenty-two isolates, the most mucoid group, were selected for further examinations. All of them were identified as Streptococcus salivarius. The GT activity of some isolates, determined by the measurement of the incorporation of radioactivity into polysaccharides and oligosaccharides from [ $\left.U-1{ }^{14} \mathrm{C}\right]-$ sucrose, was considerably higher than that of a reference strain of $S$. salivarius (HHT). The major polysaccharide produced by the GTs of fecal isolates seemed to be fructan because the color of the spot produced on a thin-layer-chromatography plate, which did not migrate, was similar to that of levan. GTs acted in the contents of small intestine collected from rabbits, showing the possibility that they may normally act in the small intestine, where a large number of streptococci live as a member of normal flora.
\end{abstract}

Key words : feces; fructan; glycosyltransferase; Streptococcus salivarius

Bacterial dextransucrase and levansucrase constitute a unique group of glycosyltransferases (GTs), which are secreted extracellularly or associated with the surface of bacterial cells and produce glycan from sucrose at a single step without any intermediate metabolite such as sugar nucleotides. In dental caries, GTs produced by Streptococcus mutans and S. sobrinus are the most important pathogenic factors (3). GTs produce water-insoluble glucan, which attach the bacterial cells to the tooth surface, resulting in the formation of dental plaque followed by dental caries. For the bacteria elaborating GTs themselves, glycans produced by the enzymes are considered to act as reservoirs of carbon source under starvation (8). GTs appear to be advantageous for these bacteria to produce glycan quickly outside the cells.

Streptococci other than $S$. mutans or $S$. sobrinus are also known to elaborate GTs (4). Glucan-producing species include S. bovis, S. sanguis, S. mitior, S. rattus, $S$. cricetus, $S$. ferus and $S$. oralis, while $S$. salivarius produces fructan $(2,7)$. However, the ecological state of them has been insufficiently known.

Especially, the ecology of GT-elaborators in the intestinal microflora remains to be further clarified. In a previous study, as a result of a surveillance of GT- 
elaborators from human and animal feces, the authors isolated three strictly anaerobic strains producing GTs from animals (6). In this study, the authors carried out the isolation and characterization of GT-producing streptococci from human feces and then discuss the ecological significance of the enzymes in the human intestine.

\section{MATERIALS AND METHODS}

Bacterial strains. Strain HHT of $S$. salivarius (13) was given by H. Akama, National Institute of Health, Tokyo. S. bovis (JCM 5672) and Leuconostoc mesenteroides (ATCC 10830) (14) were obtained from Japan Collection of Microorganisms (Wako) and American Type Culture Collection (Rockville, U.S.A.), respectively.

Isolation procedure of GT-producing streptococci. The isolation was carried out as a part of the previous surveillance $(6)$. For primary culture, TATAC plate medium (9) supplemented with $1 \%(\mathrm{w} / \mathrm{v})$ sucrose was used. Among 15 fecal samples collected from 13 individuals (Table 1), ca. 80,000 colonies were examined for their colony appearance on the sucrose-containing TATAC plates after the anaerobic incubation for 2 days at $37^{\circ} \mathrm{C}$ by the steel-wool method (11). Highly mucoid colonies were subcultured on a pair of confirmatory plates composed of Brain Heart Infusion Agar (Difco Laboratories, Detroit, U.S.A.) supplemented with or without $4 \%(\mathrm{w} / \mathrm{v})$ sucrose and were incubated at $37^{\circ} \mathrm{C}$ for 2 days anaerobically. Colonies

Table 1. Isolation of strains showing mucoidicity on selective medium

(TATAC) containing sucrose from human feces

\begin{tabular}{|c|c|c|c|c|}
\hline Specimen & $\begin{array}{c}\text { Age } \\
(\mathrm{Sex})\end{array}$ & $\begin{array}{c}\text { Number of } \\
\text { observed } \\
\text { colonies } a)\end{array}$ & $\begin{array}{c}\text { Number of } \\
\text { highly } \\
\text { mucoid } \\
\text { colonies }^{b)}\end{array}$ & $\begin{array}{l}\text { Selected colonies } \\
\text { for further } \\
\text { characterization }^{c)}\end{array}$ \\
\hline 1 & $38(\mathrm{M})$ & 697 & 1 & 0 \\
\hline 2 & $27(\mathrm{M})$ & ca. $\quad 5,000$ & 7 & 0 \\
\hline 3 & $37(\mathrm{M})$ & ca. $\quad 6,000$ & 48 & 2 \\
\hline 4 & $28(\mathrm{~F})$ & ca. $\quad 7,000$ & 0 & 0 \\
\hline 5 & $26(\mathrm{~F})$ & ca. 7,500 & 2 & 0 \\
\hline 6 & $29(\mathrm{~F})$ & ca. 3,500 & 20 & 0 \\
\hline 7 & $36(\mathrm{M})$ & ca. 10,000 & 51 & 5 \\
\hline 8 & $29(\mathrm{~F})$ & ca. 4,500 & 5 & 0 \\
\hline 9 & $32(\mathrm{M})$ & ca. 5,000 & 24 & 6 \\
\hline 10 & $27(\mathrm{~F})$ & ca. 9,000 & 6 & 0 \\
\hline 11 & $47(\mathrm{M})$ & ca. $\quad 6,000$ & 6 & 0 \\
\hline 12 & $38(\mathrm{M})$ & ca. 3,000 & 30 & 8 \\
\hline 13 & $0(\mathbf{F})$ & ca. 2,300 & 0 & 0 \\
\hline 14 & $0(\mathbf{F})$ & ca. $\quad 5,000$ & 2 & 0 \\
\hline 15 & $27(\mathrm{M})$ & ca. $\quad 5,500$ & 17 & 1 \\
\hline Total & & ca. 80,000 & 219 & 22 \\
\hline
\end{tabular}

a) Observed on the primary TATAC plates.

b) Observed on the confirmatory BHI plates.

c) Assays (TLC or PPC) of GT activity. 
showing mucoidicity only on the sucrose-containing $\mathrm{BHI}$ plate were characterized further.

Identification of isolates. Gram's staining, catalase reaction, and oxidationfermentation (OF) test of glucose were carried out according to conventional methods. Specific identification of streptococci was carried out by using Minitek Kit for gram-positive cocci (BBL, Baltimore, U.S.A.) (5).

Detection of $G T$ in culture supernatant of isolates by thin-layer chromatography (TLC). One colony of each isolate was inoculated into $5 \mathrm{ml}$ of $\mathrm{BHI}$ and incubated at $37^{\circ} \mathrm{C}$ for $24 \mathrm{hr}$ without shaking. Then, $200 \mu \mathrm{l}$ of aqueous solution of $20 \%(\mathrm{w} / \mathrm{v})$ sucrose and $20 \mu \mathrm{l}$ of aqueous solution of $10 \%(\mathrm{w} / \mathrm{v})$ sodium azide were added to $1.8 \mathrm{ml}$ of the broth culture. This reaction mixture was incubated at $37^{\circ} \mathrm{C}$ for $24 \mathrm{hr}$. One microliter of the supernatant of the mixture, obtained by the centrifugation at 10,000 rpm for $5 \mathrm{~min}$, was spotted onto a thin-layer chromatography (TLC) silica-gel plate (60 F-254, Merck, Rahway, U.S.A.) and separated in ascending chromatography for $60 \mathrm{~min}$ in the solution system of 2-propanol : acetone : $0.1 \mathrm{~m}$ lactic acid $(4: 4: 2, \mathrm{v} / \mathrm{v})$. The plate was dried in air, sprayed with diphenylamine reagent, and heated at $95^{\circ} \mathrm{C}$ for $5 \mathrm{~min}$. Spots showing various colors were identified by comparing with standard sugars, which were purchased from Sigma Chemical Co. (St. Louis, U.S.A.).

Quantitative assay of $G T$. The reaction mixture was composed of $5 \mu \mathrm{l}$ of crude enzyme, $10 \mu \mathrm{l}$ of $0.1 \mathrm{~m}$ acetate buffer $\left(\mathrm{pH} 6.0\right.$ ) containing $0.2 \%(\mathrm{w} / \mathrm{v}) \mathrm{NaN}_{3}$ and $10 \mathrm{~mm} \mathrm{NaF}$, and $5 \mu \mathrm{l}$ of $8 \%(\mathrm{w} / \mathrm{v})$ sucrose solution containing $0.1 \mu \mathrm{Ci}\left[U-{ }^{14} \mathrm{C}\right]-$ sucrose. The mixture was incubated without shaking at $37^{\circ} \mathrm{C}$ for $12 \mathrm{hr}$. Two microliters of the mixture were sampled and spotted onto Toyo No. 514A filter paper and separated by ascending paper chromatography (PPC) using a solvent system of 2-propanol : acetone : $0.1 \mathrm{~m}$ acetic acid $(4: 4: 2, \mathrm{v} / \mathrm{v})$. In this system, sucrose, glucose and fructose migrated at the distances of $20-50 \mathrm{~mm}$ from the origin after one-hour separation. Radioactivity on the filter cut out was detected by a liquid scintillation counter (Packard, Model 4530). Radioactivity on the origin of chromatography was counted as that of polysaccharides produced from sucrose, and that at the distance from 5-20 mm was counted as that of oligosaccharides.

Effect of the contents of rabbit small intestine. After two male adult Japanese-White rabbits were killed by exsanguination, the contents of whole small intestine were pooled. Aliquots of the homogenates were preserved at $-80^{\circ} \mathrm{C}$ until use. The enzymatic assay of GT in the presence of intestinal contents was carried out by measuring the incorporation of ${ }^{14} \mathrm{C}$-sugar into polysaccharides and oligosaccharides, which is described above. Culture supernatant of isolates 03122 and 09105, and strain HHT was concentrated by $50 \%$ ammonium sulfate precipitation and dialyzed against saline at $4^{\circ} \mathrm{C}$ for 2 days. Enzyme solution was concentrated to about 15 times that of the initial preparation. The reaction mixture was composed of $5 \mu \mathrm{l}$ of crude enzyme, $5 \mu \mathrm{l}$ of diluted rabbit intestinal contents, $5 \mu \mathrm{l}$ of $0.1 \mathrm{~m}$ acetate buffer $(\mathrm{pH} 6.0)$ and $5 \mu \mathrm{l}$ of $20 \%(\mathrm{w} / \mathrm{v})$ sucrose containing $\left[U-{ }^{14} \mathrm{C}\right]$ sucrose and incubated at $37^{\circ} \mathrm{C}$ for $12 \mathrm{hr}$. Samples were collected periodically and $2 \mu \mathrm{l}$ of them were spotted onto the filter paper, followed by the PPC. Radio- 
activity of polysaccharides or oligosaccharides was counted by the method described above.

\section{RESULTS}

From 15 fecal samples collected from 13 individuals, ca. 80,000 colonies were examined for their colony appearance on sucrose-containing TATAC plates after the anaerobic incubation for 2 days. Among them, 219 colonies were confirmed as to the mucoid property on the confirmatory plates. The frequency of isolation varied from person to person (Table 1). Also, there was no tendency of frequent isolation in any age or sex. In particular, 22 isolates showed the most remarkable mucoidicity. The great majority of them showed different colony appearance from that of Streptococcus bovis or Leuconostoc mesenteroides, a watery one. The isolates

Table 2. Bacteriological characterization of isolates showing severe mucoid appearance

\begin{tabular}{|c|c|c|c|}
\hline \multirow{2}{*}{$\frac{\text { Properties }}{\text { Gram's stain }}$} & \multicolumn{2}{|c|}{$\begin{array}{c}22 \text { isolates } a) \\
\text { (Number of positive) }\end{array}$} & \multirow{2}{*}{$\begin{array}{c}\begin{array}{c}\text { S. salivarius } \\
\text { strain HHT }\end{array} \\
+\end{array}$} \\
\hline & + & $(22)$ & \\
\hline Morphology & coccus in chain & $(22)$ & coccus in chain \\
\hline $\mathrm{OF}$ & $\mathrm{F}$ & $(22)$ & $\mathrm{F}$ \\
\hline Catalase & - & $(0)$ & - \\
\hline \multicolumn{4}{|c|}{ Minitek system for gram-positive coccus (BBL) } \\
\hline Arginine & - & $(0)$ & - \\
\hline Arabinose & - & $(0)$ & - \\
\hline Galactose & + & $(22)$ & + \\
\hline Inulin & + & $(20)$ & + \\
\hline Lactose & + & $(22)$ & + \\
\hline Esculin & + & $(22)$ & + \\
\hline Maltose & + & $(22)$ & + \\
\hline Mannitol & - & $(0)$ & - \\
\hline Mannose & + & $(22)$ & + \\
\hline Raffinose & $\mathrm{d}$ & (17) & + \\
\hline$\beta$-Galactosidase & + & (22) & + \\
\hline Salicin & + & $(22)$ & + \\
\hline Sorbitol & - & (2) & + \\
\hline Trehalose & $\mathrm{d}$ & (8) & - \\
\hline Glucose & + & (19) & + \\
\hline Nitrate reduction & - & $(0)$ & - \\
\hline Voges-Proskauer & - & $(0)$ & - \\
\hline Phosphatase & $\mathrm{d}$ & $(15)$ & - \\
\hline Hippurate & - & $(0)$ & - \\
\hline Pyroglutamic acid & - & $(0)$ & - \\
\hline Leucine & - & $(0)$ & - \\
\hline \multicolumn{4}{|c|}{$\begin{array}{c}\text { Streptococcus } \\
\text { salivarius }\end{array}$} \\
\hline
\end{tabular}

a) + , more than $80 \%$ strains are positive; -, more than $80 \%$ strains are negative; d, strains more than $20 \%$ and less than $80 \%$ are positive. 


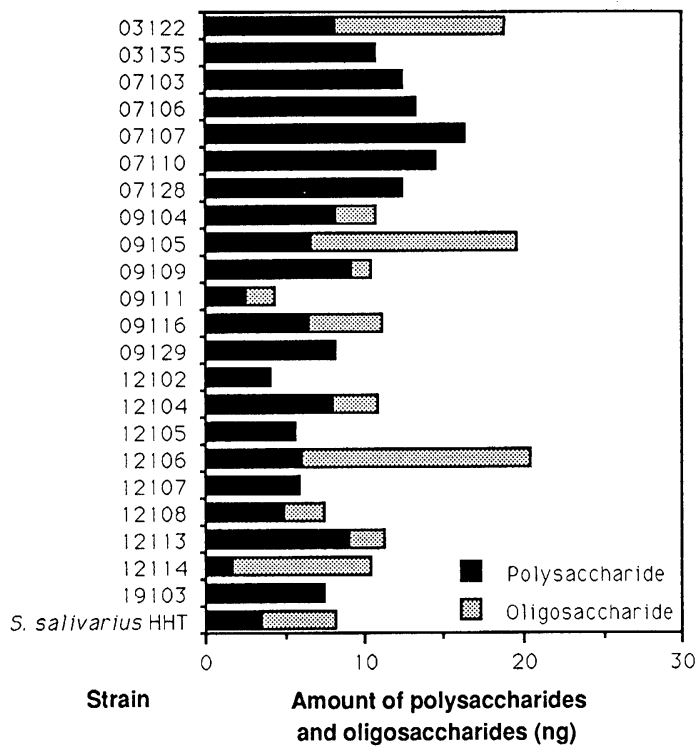

Fig. 1. Incorporation of radioactivity into polysaccharides and oligosaccharides from $[U-14 \mathrm{C}]$ sucrose.

showed more or less stickiness.

Results of bacteriological examination of the 22 isolates showed that all of them had the characteristics of the genus Streptococcus, i.e., gram-positive cocci, catalasenegative, glucose-fermenting $(\mathrm{OF}(\mathrm{O}))$ and facultative anaerobic. By using Minitek Kit for gram-positive cocci, all of them were identified as Streptococcus salivarius. The results are shown in Table 2.

Detection of GT in culture supernatant of the isolates by thin-layer chromatography (TLC) was carried out. On TLC plates, the mixture of enzyme reaction of the isolates showed distinct spot on the chromatographical origin, with the color of brown, corresponding to that of fructose or levan used as a standard. The major polysaccharide species produced by GTs of fecal $S$. salivarius is probably fructan because the color of spots produced on the TLC plate was similar to that of fructose or levan (data not shown). However, heterogeneity of enzymes between isolates may be present, as relatively faint spots of oligosaccharides were detected on TLC plates.

On TLC plates, some monosaccharides such as glucose and fructose were detected, which may indicate the presence of invertase, a sucrose-hydrolyzing enzyme (1). For quantitative GT assay, a method using radioactive sucrose as a substrate was adopted, because this method can estimate direct incorporation of monosaccharide moieties into polysaccharides. The radioactivity on the filter paper cut out was not lost by washing with absolute methanol, indicating this fraction is polysaccharides. The results of 22 isolates are shown in Fig. 1. Besides polysaccharides, radioactive spots thought to be oligosaccharides were detected in some 


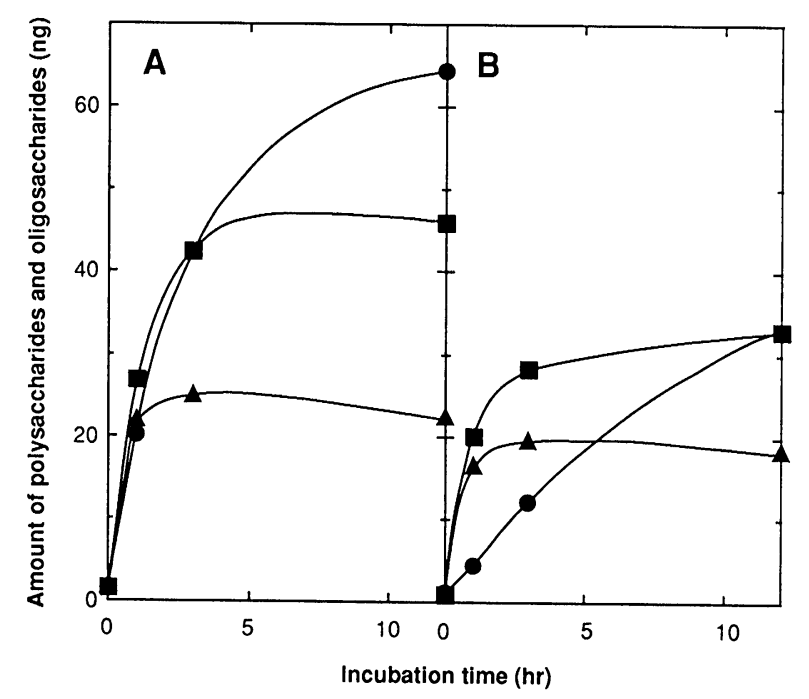

Fig. 2. Effect of the rabbit intestinal contents on the GT activities of $S$. salivarius. A, strain 03122 ; B, strain HHT. Reaction mixture was added without (-), with $5 \%$ (घ) and $12.5 \%$ ( $\Delta$ ) rabbit intestinal contents.

isolates. The strongest enzymatic activity among the isolates was considerably higher than the activity of the GT of $S$. salivarius HHT, used as a standard.

The effect of rabbit intestinal contents on GT activity is shown in Fig. 2. Because sufficient GT activity of the isolate 03122 was detected in the supernatant $(41.0 \%)$, we used its culture supernatant concentrated by ammonium sulfate precipitation as the GT source. In general, crude GT of 03122 can act in the presence of rabbit intestinal contents (Fig. 2A). While 12-hr incubation with the rabbit intestinal contents inhibited the incorporation of ${ }^{14} \mathrm{C}$-sugar into polysaccharides and oligosaccharides, 3-hr incubation rather enhanced incorporation. Similar results were obtained by the crude enzyme of isolate 09105 (data not shown). Enzyme of strain HHT showed similar results, but when measured at $12 \mathrm{hr}$, inhibition of GT activity was relatively small (Fig. 2B). Thus, behavior of enzymes was somewhat different between the isolates (03122 and 09105) and the standard strain (HHT).

\section{DISCUSSION}

Are GTs of $S$. salivarius functional in human intestine? Many streptococcal species live in the middle (jejunum) and lower (ileum) part of human small intestine at the cell number of $1 \times 10^{4}$ to $1 \times 10^{6-7}$ per gram of contents, respectively (9). Because $S$. salivarius is a member of normal fecal flora, it is likely that this species seems to live in the small intestine (15). The substrate, i.e., sucrose, is hydrolyzed into monosaccharides with the host enzyme on the wall of small intestine and ab- 
sorbed, resulting in the decrease in the amount of sucrose at the lower part of small intestine and large intestine. In this study, we demonstrated that the strongest producer of GTs in the fecal isolates is $S$. salivarius and showed that the production of polysaccharide by GTs of fecal isolates of $S$. salivarius could occur in the presence of rabbit intestinal contents.

Polysaccharide produced by GTs of fecal $S$. salivarius is probably fructan because the color of spots produced on the TLC plates was similar to that of fructose or levan. Strain HHT of S. salivarius was shown to produce levan-type fructan (2). However, further studies are required to characterize enzymes of fecal strains of $S$. salivarius to compare with the enzymes of oral strains. Also physicochemical characterization of produced fructan(s) remains to be studied in detail.

Pabst (10) postulated the three significances of extracellular levan produced by Actinomyces viscosus in the pathogenesis of periodontal disease. (i) Levan may be an important structural polysaccharide in dental plaque. (ii) Levan may provide a reservoir of carbohydrate for the growth of oral microbes. (iii) Levan may trigger immunological injury, since levan from other organisms has been shown to be mitogenic for human B-lymphocytes and to activate complement by the alternative pathway. Similar possibilities, except (i), could be postulated also for streptococcal polysaccharide in the small intestine. Levanase of $S$. salivarius may play a role in the utilization of produced levan at the lower part of intestine $(1,12)$.

Although all the isolates producing GT were identified as $S$. salivarius, their properties were not identical with that of HHT. In the presence of intestinal contents, GT activity of fecal isolates was enhanced only slightly when determined after 3-hr incubation. On the other hand, GT of HHT was considerably enhanced in the same condition. These results indicate that fecal isolates produce GT, being different from that of strain HHT of $S$. salivarius. Although strains of $S$. salivarius are frequently isolated from human feces (15), further characterization should still be carried out to compare fecal and oral strains of $S$. salivarius.

In conclusion, the authors isolated glycosyltransferase-elaborating $S$. salivarius from human feces. The fructan-producing ability is perhaps retained in the small intestine, where streptococci live predominantly.

Acknowledgments. The authors thank Mr. M. Saito for the expert technical assistance. We are very grateful to Ms. M. Takagaki, Sagami Chemical Research Center, for her valuable help.

\section{REFERENCES}

(1) Chassy, B. M., J.R. Beall, R.M. Bielawski, E.V. Porter, and J.A. Donkersloot. 1976. Occurrence and distribution of sucrose-metabolizing enzymes in oral streptococci. Infect. Immun. 14: 408-415.

(2) Ebisu, S., K. Kato, S. Kotani, and A. Misaki. 1975. Structural differences in fructans elaborated by Streptococcus mutans and Str. salivarius. J. Biochem. 78: 879-887.

(3) Hamada, S., and H.D. Slade. 1980. Biology, immunology, and cariogenicity of Streptococcus mutans. Microbiol. Rev. 44: 331-384.

(4) Hardie, J.M. 1986. Genus Streptococcus Rosenbach 1884, 22 AL, p. 1043-1071. In N.R. Creig and 
J.G. Holt (eds.), Bergey's manual of systematic bacteriology, Vol. 2, The Williams \& Wilkins Co., Baltimore.

(5) Holloway, Y., M. Schaareman, and J. Dankert. 1979. Identification of viridans streptococci on the Minitek miniaturized differentiation system. J. Clin. Microbiol. 32: 1168-1173.

(6) Inoue, A., K. Yazawa, K. Kondo, T. Fujisawa, and T. Mitsuoka. 1989. Isolation of bacteria elaborating glycosyltransferase from the feces from human and animals. Bifidobacteria Microflora 8: 95-99.

(7) Long, L.W., S.S. Stivala, and J. Ehrlich. 1975. Effect of $\mathrm{pH}$ on the biosynthesis of levan and on the growth of Streptococcus salivarius. Arch. Oral Biol. 20: 503-507.

(8) Manly, R.S., and D.T. Richardson. 1968. Metabolism of levan by oral samples. J. Dent. Res. 28: 1080-1086.

(9) Mitsuoka, T. 1980. The world of intestinal bacteria-the isolation and identification of anaerobic bacteria; a color atlas of anaerobic bacteria (English title given), Sobunsha, Tokyo.

(10) Pabst, M.J. 1977. Levan and levansucrase of Actinomyces viscosus. Infect. Immun. 15: 518-526.

(11) Parker, C.A. 1955. Anaerobiosis with iron wool. Aust. J. Exp. Biol. Med. Sci. 33: 33-38.

(12) Takahashi, N., F. Mizuno, and K. Takanori. 1983. Isolation and properties of levanase from Streptococcus salivarius KTA-19. Infect. Immun. 42: 231-236.

(13) Terleckyj. B., N.P. Willet, and G.D. Shockman. 1975. Growth of several cariogenic strains of oral streptococci in a chemically defined medium. Infect. Immun. 11: 649-655.

(14) Tsuchiya, H.M., N.N. Hellman, and H.J. Koepsell. 1953. Factors affecting molecular weight of enzymatically synthesized dextran. J. Am. Chem. Soc. 75: 757-758.

(15) Watanabe, T., H. Shimohashi, Y. Kawai, and M. Mutai. 1981. Studies on streptococci. I. Distribution of fecal streptococci in man. Microbiol. Immunol. 25: 257-269. 